\title{
ROAD SIGNS: WALKING AMONG SHAPES AND COLORS
}

\author{
Mariangela Zedda ${ }^{1}$, Claudia Piras ${ }^{2}$, Francesco Pinna $^{3}$ \\ ${ }^{1} \mathrm{PhD}$ student, ${ }^{2} \mathrm{PhD},{ }^{3}$ Professor, Department of Civil, Environmental Engineering and Architecture, University of \\ Cagliari,Italy,mar.zedda@unica.it,cpiras@unica.it,fpinna@unica.it
}

\begin{abstract}
Road signs provide information through the combination of three elements: shape, color and pictogram. The current road signs are designed to ensure safety but are designed for adults and not for children. Kids are not small adults and they have a different perception of reality. While planning of home to school pedestrian routes, it is necessary to create road signs that are comprehensible for children aged 6-14. Therefore the elements of road signs were studied individually. The study revealed that both the knowledge of geometric shapes and colors perception are closely related to age and culture and to the teachings which children are submitted to. In fact, some geometric shapes, like the circle, are perfectly recognized from childhood and others less so, such as the rectangle. Moreover children are attracted to basic colors but they are aware of the existence of pastel colors only with teaching. Finally, pictogram must represent a simple symbol that is easily recognized especially from far away. The message generated by the three elements is understandable by children. In fact, children overcome the phase of syncretism after the age of 6, which corresponds to the phase in which children are unable to analyses and syntheses. The result of the research has allowed designing road signs that are able to ensure the safety of home to school routes. A road signs study was conducted for Cagliari's project on road safety. This project involves the use of questionnaires to verify if the road signs are properly designed.
\end{abstract}

Keywords: home to school routes, road signs, color, shape.

\section{INTRODUCTION}

Home to school routes offer children the opportunity to move independently and safely in their daily life environment, the town. They represent a model of alternative mobility to the chaotic and excessive use of private vehicles. Also, the home to school routes contribute to the reduction of vehicular traffic during the morning rush hour, and allow children to socialize and at the same time to make physical exercise and to have greater awareness of urban land. The realization of home to school routes permits to resize the city making it "fit for children". The reorganization of road sections and the adoption of traffic calming measures do not guarantee total safety. Therefore road signs play a very important aspect because they must give guidance and at the same time indications so that the planned route appears recognizable and safe especially for children. The current road signs are projected to ensure safety but they are designed for adults and not for children. Kids are not small adults and have a different perception of reality. For this reason, in order to design effective road signs, you must understand how children perceive shape, color and pictogram and if they are able to understand the message. This paper is divided into two parts. The first part analyzes the elements that constitute the road signs such as shape, color and pictogram while the second part describes the design of new road signs.

\section{ROAD SIGNS STUDY: SHAPE, COLOR AND PICTOGRAM}

\subsection{Perception of the Geometric Shape}

The shapes of road signs are geometric shapes. In Italy the most common shapes are circle, rectangle, triangle and hexagon. At what age children can recognize geometric shapes? According to the theory developed by Dina van HieleGeldof and Pierre van Hiele [1-2], this depends on the experiences and lessons to which children are subjected. The theory is based on 5 levels of development of geometric thought. The hierarchy of levels spans from nursery school to university. The first level is called "Visual" in which children are able to recognize the shapes presented to them but they cannot create mental images. They use some visual prototypes to identify the shapes. They can say, for example, that "this is rectangle because it looks like a door." So they fail to formulate definitions because they have not yet learned the properties and attributes of shapes. In the second level, called "descriptive - analytic", children describe the figures based on the geometric properties, the images lose importance compared to their attributes, for example they can say that "this figure is a square because it has four equal sides" but cannot say that "it is square because it has equal angles". The third level is "informal deductions" or of Euclidean geometry in which children observe the various relationships between figures from the logical point of view and learn the technical terminology. So they can classify shapes with logical 
arguments. In the fourth level, deductive or formal logic, the students are able to distinguish between a proportion and its inverse. They understand demonstrations, postulates, axioms and theorems. In the last level there is "geometrical rigor", students learn no- Euclidean geometry and are able to compare different systems of axioms.

Thereafter Clements and Battista [3] have taken up and updated this theory. They have added a level, called prerecognition, which precedes the visual one. In this level children recognize shapes but are unable to classify or to draw them.

This model has some properties. The levels are sequential and the progress from one level to the next does not depend on age but on instructional experience. Also the object of interest to a level becomes the object of study in the next level. Each layer is characterized by a specific use of language that can be considered appropriate within that level and may be improved only in the next level.

Some researchers have performed a test in which they were interviewed 128 children, ages 3 to 6 . The test consisted in the identification of some geometric shapes (circle, square, triangle and rectangle) and its recognition even if in different orientations. The results showed that the most easily recognized figure is the circle (only $4 \%$ answered incorrectly), although children under 6 years also identified the ellipses like circle, the square has been recognized correctly by $87 \%$; the triangle was identified as such by only $60 \%$ and finally $54 \%$ of the tested children recognized the rectangle.

\subsection{The perception of Color}

Color is the central element of the visual code that facilitates the perception of reality. It has the ability to capture the attention of the people, to provide information and to have suggestive power. Its properties are used in different fields. Color is used in advertising to persuade consumers. Color is a determining factor in purchasing decisions. Also the choice of color makes the brand recognizable through its typical colors. In addition, colors are used in websites to enhance the graphics, layout and content support, the navigation menu. In architecture colors are used to change the perception of proportion, to hide or highlight special architectural elements. Finally, the colors are also used in medicine as a remedy for some diseases. In fact, in chromo therapy every color is associated with psychological characteristics of the individual. What are the physical properties that allow the color to influence the individual?

Color is defined by three parameters: hue, saturation and brightness. The hue identifies colors and it allows them to be defined as red, yellow, blue and green. The saturation identifies the intensity, brilliance and purity of color. The brightness expresses light intensity, quantifies the percentage of black and white in the perceived color, so the degree of light and dark. Colors are classified according to the "Itten's color circle"[4] in which in the center of the circle there is a triangle that contains the three basic or primary colors which are red, yellow and blue. From the mixture of these, in equal parts, you get the secondary colors that are green, purple and orange, and they are located around the central triangle. Finally, in the outer circle there are 12 tertiary colors obtained by mixing pure colors. Also according to Itten to get complementary colors you have to choose a color and follow the opposite one. Complementary colors have the characteristics to enhance and strengthen each other's brightness. The colors possess own temperature and are divided into warm, cool and neutral (or achromatic) because they convey different feelings and bring to mind particular images and situations. In fact they are called hot, because they evoke sensations of heat, for example yellow is associated to the sun, the red to fire. Conversely, cool colors evoke feelings of cold as the sky, the sea, the snow, the ice. Johannes Itten identifies 7 contrasts with which you can control both the intensity and brilliance of the colors, and get visual effects and perspective effects. Among the latter, chrome agreements are able to produce optical effects or tonal combinations to attract the attention of the observer. Another property of color is to be able to create a sense of movement. When the values of a color leap quickly from very high to very low, it is to create a feeling of excitement and movement. Also the use of repeated elements can create the illusion of movement. The visual rhythm is perceived through the eyes and is created by repeating positive spaces alternating with negative spaces. There are 5 types of rhythm: random, regular, alternating, flowing and progressive.

\subsection{Color Psychology}

Psychologists who are very interested in the study of colors argue that colors evoke emotional or psychological effects. According to the theories of the authors [5-6], color-emotion associations are pleasant or unpleasant. Generally, the warm colors are aggressive, stimulants or positive, while cold ones are tranquil, serene. In psychology, the color becomes a sensation:

- Red has the characteristic of increasing the heartbeats causing a adrenaline reaction. Along with the yellow it is the first to be processed by brain. It is a color that conveys impulsiveness, speed, determination, danger. It is said to be the first to be perceived by children.

- Blue is the color that the human eye perceives even at great distances. It transmits calmness, tranquility and serenity.

- Yellow increases the ability to concentrate and the creation of serotonin (regulates good mood). If it is used incorrectly, it might not be clearly visible. It stimulates reflection and memorization and transmits creativity, warmth, energy, optimism and curiosity. 
- Green is the color that the brain process more easily and has a calming effect. It conveys harmony, peace, nature, balance, stability.

- Orange increases the instinct to act and conveys movement and energy without overcoming the observer. It can help to change attitude and transmits energy, accessibility, enthusiasm.

- Purple stimulates troubleshooting. Notify mystery, imagination and creativity.

The psychiatrist Francesco Simeti [7] has conducted some research along with a team of experienced psychotherapists and they focused on the analysis of all the components that contribute to create the image and subjective expression of colors on an emotional level. In particular, he studied how the color is perceived by children. The experimental research has been performed on a group of 500 children of different age groups and on a group of adults. According to Professor Simeti, color perception is closely linked to the child's age and has always lived in connection with the five senses. Furthermore he argues that if in the first years of life the color is and remains a natural perception, with the growth people are influenced by the teachings and culture. So the perception of color is not subjective anymore. Besides he claims that natural colors for example shades like flame red, grass green, blue and white, derive from the direct contact of the child with reality and for that reason they are universal. Then there are some colors more related to some cultural traditions, for example in the Far East glaucous and sepia are also considered fundamental. Children develop from early childhood a relationship with colors that keep them well away from the contact with nature. However adults impose the colors of clothing and toys on children. Infants have a relationship with colors that comes from the contact with nature. From early childhood, children begin to experience the colors of a reality created by the projections of the fantasies of adults that correspond neither to nature nor to mental images. Research has shown that children, regardless of gender, are attracted by the basic colors, bold hues and not to pastel shades. So children prefer bright colors and they do not like the nuances and refuse pastel color.

\subsection{Pictogram and Syncretism}

Pictogram is a universal language, a writing form in which the graph sign indicates an object, an elementary idea. Often it is used to communicate quickly and incisively without the use of the words. Pictogram has the aim to be self-explanatory, in other words, the symbol must be easily associated with what it wants to represent. Symbols must be simple in their graphical representation, culturally neutral and the most universally known. Children understand the meaning of the most common symbols because since childhood they are in close contact with the figures that are useful for easy learning. In fact, the child is encouraged to learn and communicate orally through a system of communication through the exchange of images. It is during childhood that, for example, through symbols, they learn about the shapes that can be animals, objects, letters, nouns, verbs. The key problem is whether the child of 6-14 years, understands the meaning of the pictogram depicted in the road sign. Piaget [5] has performed studies about infantile mentality. In particular, he identifies in child development the syncretism phase in which the child is unable to analysis and synthesis. At the perceptual level, the syncretism is the inability which leads the child to grasp figure or object, everything or the most eye-catching. The perception of the whole or parts depends on the stimulus. Children can perceive details if these are conspicuous or may perceive the whole but in a confused way finding it difficult to identify the details that constitute it. This phase is characteristic of the childhood; you witness the overcoming of syncretism from 6 years onwards.

\section{ROAD SIGNS PROJECT}

The project of vertical road signs initially focused on finding a central thread that tied the four elements that constitute it [8]. Based on the above, it can be said that the only element that is able to create a balance and join the elements is just the color. So colors can be a double edged sword because if they are not used properly, they can create imbalance so as to give greater importance to an element rather than another. Children should be stimulated to look at the sign, therefore it was decided to use the color properties with which you can create movement for the metal support. This can be achieved by alternating stripes of black and white with oblique lines, or sequence of elements. They were chosen black and white because any other color would to draw attention only to the support element. The figure (Fig-1) shows 4 options: on the first alternating horizontal stripes are used and their width becomes narrower with height, on the second, it takes curvilinear alternating stripes of equal thickness, on the third the same criterion of the second is followed but with variable thickness which decreases with height, and the last, however, is like the third but the texture is more dense. So the support establishes a visual path that guides the viewer to look at the sign.

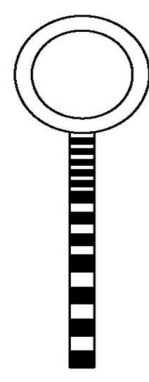

(a)

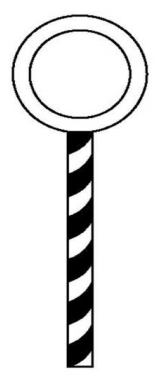

(b)

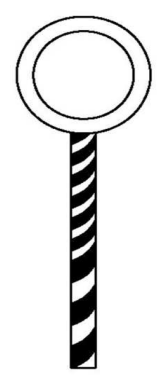

(c)

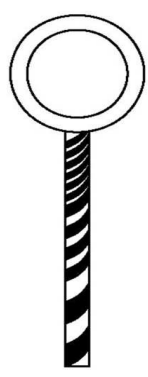

(d)
Fig-1: Examples of metal support 
The sign must express a visual message that is direct and easy to read in its entirety. In order to be perceived clearly and to be balanced in the composition, it was decided to use color contrast. The circular shape is preferred for the signal that indicates the beginning of the route (Fig-2), since it is the most easily recognized by children. Then, the circle has the characteristic of producing a strong centralizing effect on perceptual modalities of the elements that constitute the composition. In addition, if the circle enters into relationship with a system of free lines or contains simply an image, it generates harmonious effect and balance. A simple template was chosen as pictogram which represents a student (child with backpack). Since it must be visible from a great distance, the contrast chosen was the one between colors warm-cool such as blue-red, blue for the background and red for the pictogram. This contrast appears to be the most appropriate because blue is clearly visible from great distances, as well as the red.

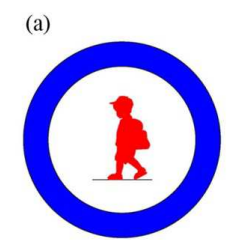

Road signs which indicate the beginning of route

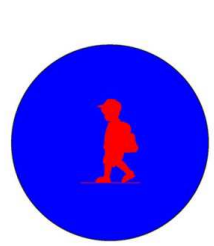

(d)

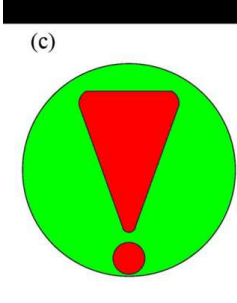

Pedestrian Crossing
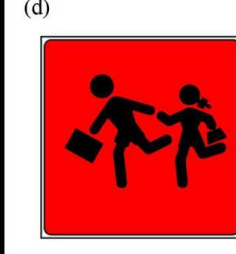

Road signs that indicate the end of routes
Fig-2: Road sign

The figure (Fig-2a) shows two options, the first is the one used in the design. The sign on the left was chosen because white causes an optical effect that makes the pictogram look bigger. In fact, if you look at the figure on the right, the child depicted looks more slender. Regarding the indication of the parking (kiss and ride) near the beginning of the route, a sign with square shape is preferred to be used (Fig-2b). The clip art used for the symbol depicts a stylized green car on red background. This contrast of complementary colors gives to sign stability between shape and message to the sign. About pedestrian crossings, the idea was to put a sign next to the pedestrian crossing that induces children to give more attention (Fig-2c). In this case, for the reasons described before, the shape that characterizes the sign is the circle, while for the pictogram we opted for the exclamation point. The choice of this symbol was dictated by its traditional use. Indeed, it is often used in novels and comics, or in publications for children to indicate threat or danger, or to draw the attention to something. The road sign designed to indicate the end of the route (Fig-2d) and therefore the arrival at the school has a square shape and the pictogram represents the template of two schoolchildren with a bag. The preference of this pictogram is due to the fact that in Italy the same symbol is used to indicate that a school is near, and then an image is known to children. In addition, since there have been cases in which the home to school route is common to two or more schools; it was decided to associate a color to each school. So for this reason, the background signal varies according to the school of reference.

\subsection{Road Surface Marking}

Road surface marking plays an important role because it must especially indicate the route that children must follow in order to get to school. For this reason it must be clear, easily readable and designed not to create confusion. The predominant color of the marking is yellow. This color was chosen both because it is loved by children of the considered age group, and for its psychological properties as it increases the ability to concentrate and it is thought-provoking. The beginning of the route is represented by a yellow circle with the symbol of two "big feet" inside (Fig-3).

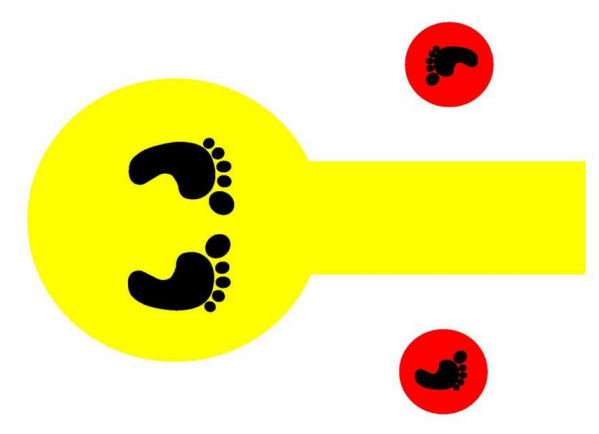

Fig-3: Horizontal marking which indicates the beginning of route.

The use of the feet as a symbol is not accidental. Indeed, this symbol is often used in illustrations for children for example in comics to represent the activity of walking or the possibility of movement. In addition, the rounded lines of the image capture the attention of the child. The route that the students must follow consists of a yellow line the sides and its are depicted with circles with the "little feet" inside. The depiction of the feet indicates both the direction of home to school and the way school to home. In addition, the background of the circles represents the color of the school which the student attends. 
(a)

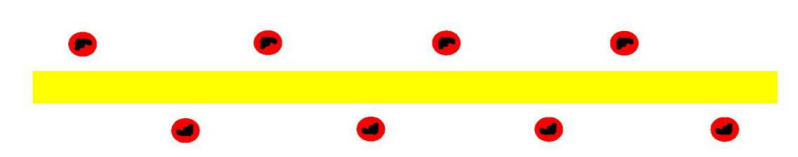

(b)

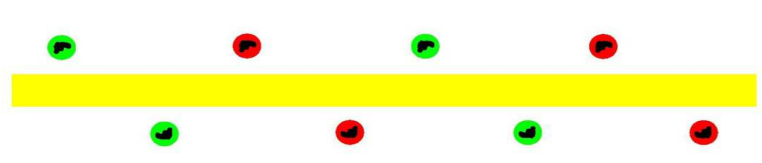

Fig-4: Home to school routes

If more schools have the same route, more feet will be depicted with the background color that represents the school. For example "Figure 4a" shows the route of a school to which the color red is associated. "Figure $4 b$ " shows the route of two schools: red school and green school. The students of the red school follow the feet with red background, while the students of the green school follow the route indicated by the feet on green background. Near the intersection of multiple routes, a sign is placed that indicates the way to be taken. So children are not confused which route must be followed to arrive at his school. The direction is indicated by a symbol that represents a colored pencil. The color of the pencil indicates the school.

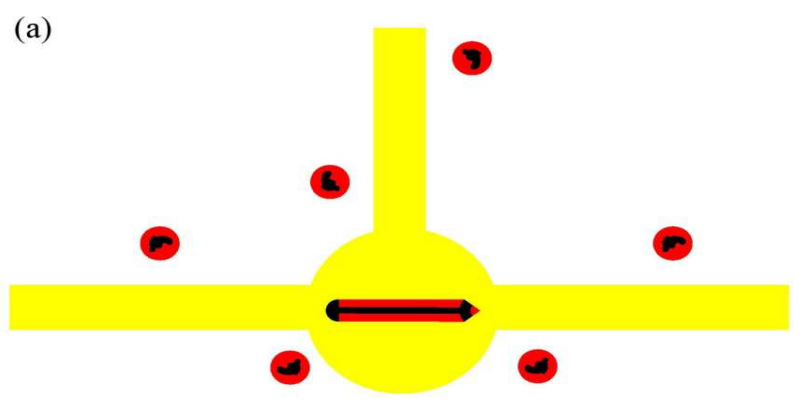

(b)

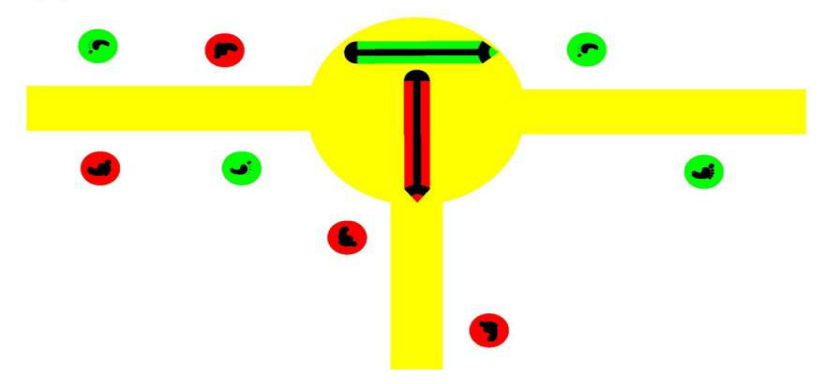

Fig-5: Examples of intersections
The figure (Fig-5) shows two cases. The first case (Fig-5a) concerns the route of a school and in particular the intersection that is characterized by three paths. The pencil is designed so that the tip indicates the correct route to take. In the second case (Fig-5b) two schools (red and green) have only part of the route that is in common. So students have to take different routes: the children of green school follow the route indicated by the green pencil, and students of the red school follow the route indicated by the red pencil. Regarding the crosswalk, signage was designed to induce children to stop and look left and right before crossing the road. They have avoided writing because children are often confused when they have to distinguish left from right (Fig-6).

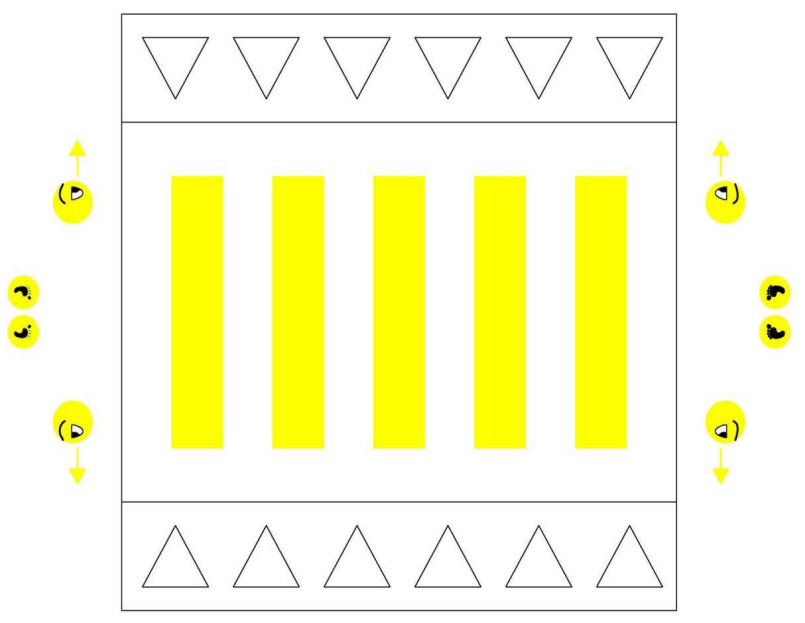

Fig-6: Pedestrian crossing

Finally, to represent the end of the route we thought of a symbol that represents the arrival at school (Fig-7). The idea was to make people remember the checkered flag used in sports, especially motor race, to indicate the end of a race (the finish line).

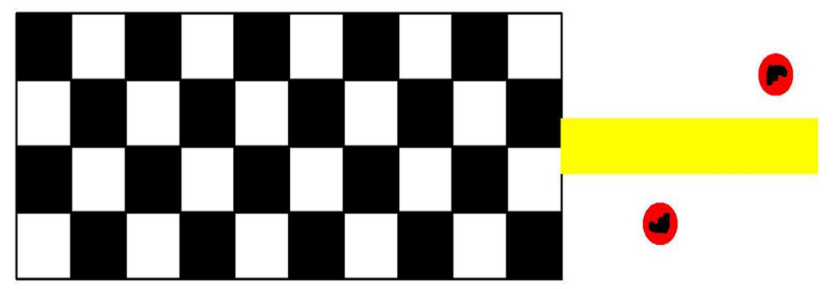




\subsection{Research Progress.}

The research unit of the University of Cagliari is carrying out the study of road signs. The next step of the study will be to know how initial hypotheses confirm the children behavior real, especially in terms of the message understanding represented by the road signs as a whole.

For this reason a road signs study will be conducted in Cagliari in Italy. The project involves the psychological study of elementary school students. In fact it will allow to know the student attitudes regarding road signs. Moreover it will be possible to know, for example, what features of the road signs as colors, shape, height, have a greater impact on their behavior and if these attitudes are adequate to type of road signs. The psychological study will consist of four phases:

- Analysis of the scientific literature on traffic psychology.

- Questionnaire construction.

- Design of a pilot study to be conducted on 50 or more children.

- Design and validity of the study on a normative sample about 500 children.

Therefore this project involves a use of questionnaire which was developed by psychologists and they will be used to verify if the road signs are properly designed. Over the coming months the questionnaires will be submitted to some elementary school children. The test results will be used to improve the design of the road signs.

\section{CONCLUSIONS}

Currently the traffic organization and the road network infrastructure is not designed for children and for preserving their safety. The home to school routes allows resizing the town and to make it fit for children through the reorganization of the road and the adoption of traffic calming measures. These actions combined with new road signs can be seen as an effective means to counter the impulsive nature of children. Road signs have the main objective to ensure safety and to send a clear message in the context of the home to school routes plays an important part because they must guide and ensure safety of the route. For this reason appropriated signage has been designed apposite for children, studying every single element of the sign and by analyzing them from the child's point of view. The research found that colors are the central thread that is able to create balance between the different elements. In addition, the rounded shape creates an effect of harmony and balance, making the sign clear in its meaning.

\section{REFERENCES}

[1]. Crowley, M. "The van Hiele Model of the Development of Geometric Thought." In M. Lindquist, ed., Learning and Teaching Geometry, K-12, 1987 Yearbook. Reston: National Council of Teachers of Mathematics, 1987.

[2]. Fuys, D., D. Geddes, and R. Tischler, "The van Hiele Model of Thinking in Geometry Among Adolescents." Journal for Research in Mathematics Education Monograph, 3. Reston: National Council of Teachers of Mathematics, 1988.

[3]. Clements, D.H., \& Sarama, J., "Young children's ideas about geometric shapes." Teaching Children Mathematics, Vol.6, 2000, pp.482-487.

[4]. Johannes Itten, "The Art of Color: The Subjective Experience and Objective Rationale of Color." John Wiley \& Sons Inc, 1974.

[5]. L. Camaioni, P. Di Blasio, "Psicologia dello sviluppo" Edizioni Il Mulino 2002.

[6]I. Romanello, "Il colore espressione e funzione", Hoepli 2002.

[7]D. Antonello, F.Simeti, S.Balliello, A. Borgo, M. De Danieli, V. Melon, M. G. Ghitti, "I colori, sensi e emozioni", CSIFRA 2002.

[8]. M. Zedda, "Forme e Colori: I percorsi casa-scuola nella città di Cagliari”, tesi presso Facoltà di Ingegneria Università degli studi di Cagliari, 2012. 